\title{
Take-Home Emergency Naloxone to Prevent Heroin Overdose Deaths after Prison Release: Rationale and Practicalities for the N-ALIVE Randomized Trial
}

\author{
John Strang, Sheila M. Bird, and Mahesh K. B. Parmar
}

\begin{abstract}
The naloxone investigation (N-ALIVE) randomized trial commenced in the UK in May 2012, with the preliminary phase involving 5,600 prisoners on release. The trial is investigating whether heroin overdose deaths post-prison release can be prevented by prior provision of a take-home emergency supply of naloxone. Heroin contributes disproportionately to drug deaths through opiate-induced respiratory depression. Take-home emergency naloxone is a novel preventive measure for which there have been encouraging preliminary reports from community schemes. Overdoses are usually witnessed, and drug users themselves and also family members are a vast intervention workforce who are willing to intervene, but whose responses are currently often inefficient or wrong. Approximately $10 \%$ of provided emergency naloxone is thought to be used in subsequent emergency resuscitation but, as yet, there have been no definitive studies. The period following release from prison is a time of extraordinarily high mortality, with heroin overdose deaths increased more than sevenfold in the first fortnight after release. Of prisoners with a previous history of heroin injecting who are released from prison, 1 in 200 will die of a heroin overdose within the first 4 weeks. There are major scientific and logistical challenges to assessing the impact of take-home naloxone. Even in recently released prisoners, heroin overdose death is a relatively rare event: hence, large numbers of prisoners need to enter the trial to assess whether takehome naloxone significantly reduces the overdose death rate. The commencement of pilot phase of the N-ALIVE trial is a significant step forward, with prisoners being randomly assigned either to treatment-as-usual or to treatment-as-usual plus a supply of take-home emergency naloxone. The subsequent full N-ALIVE trial (contingent on a successful pilot) will involve 56,000 prisoners on release, and will give a definitive conclusion on lives saved in real-world application. Advocates call for implementation, while naysayers raise concerns. The issue does not need more public debate; it needs good science.
\end{abstract}

\section{BACKGROUND}

The pilot phase of the naloxone investigation (N-ALIVE) trial is funded by the Medical Research Council in the UK and is investigating the extent to which deaths from heroin overdose in the first 12 weeks post-release from prison can be prevented by prior provision of a take-home emergency supply of naloxone. The N-ALIVE trial is the largest-ever

Correspondence: John Strang, King's College London, National Addiction Centre (Institute of Psychiatry and The Maudsley), London, SE5 8AF, UK. (E-mail: john.strang@kcl.ac.uk)

Correspondence: Shiela Bird, MRC Biostatistics Unit, Cambridge, CB2 2SR, UK. (E-mail: sheila.bird@mrc-bsu.cam.ac.uk)

Correspondence: Mahesh Parmar, MRC Clinical Trials Unit, London, WC2B 6NH, UK. (E-mail: mp@ctu.mrc.ac.uk) 
planned randomized intervention trial with a prisoner sample (or, to be more precise, at the point of release), and involves 5,600 prisoners on release in the initial pilot randomized phase, then extending to its randomized sample size of 56,000 for the full N-ALIVE trial.

The full N-ALIVE trial protocol will be published separately as a trial protocol publication (in preparation, April 2013). In this publication, we present the background scientific, clinical and political rationale, and considerations of objective, partners, interventions and measures-all crucial before the trial design and protocol can even be considered meaningfully. How have the decisions about the medication itself and the associated intervention, the study population, the period of study and the outcome measures been made?

\section{A Novel Intervention}

Take-home emergency naloxone has been proposed as a novel preventive measure to reduce the likelihood of a fatal outcome from heroin overdose amongst drug users. ${ }^{1-21}$ However, in the wider community context, reports of pilot naloxoneprescribing schemes ${ }^{10,14,15,19,21-25}$ have mostly comprised anecdotal reports of small-scale interventions with systematic follow-up rarely included, and the true nature and extent of benefit remain uncertain.

Implementation of take-home naloxone has been recommended in the UK by the Advisory Council on the Misuse of Drugs (ACMD) ${ }^{26}$ which recognized the potential contribution that take-home naloxone could make and expressed the opinion that 'as a matter of principle, naloxone should be made more widely available' accompanied by attention to implementation issues. A more recent report from $\mathrm{ACMD}^{27}$ further recommended government '... to consider naloxone's role in steps to make an impact on drug-related death rates'. However no large-scale study of actual effectiveness has yet been conducted.

This is a clear instance of where the interactive relationship between science and policy should move center stage, ${ }^{28}$ with policy asking a question of science, and with science giving a clear guiding reply.

\section{THE NEED FOR STRONGER RESEARCH AND THE ORIGINS OF THE N-ALIVE TRIAL}

The origins of the N-ALIVE trial were as a result of confluence of the drive to develop more effective methods to prevent the striking excess mortality of heroin users (excess even compared with users of other illicit drugs such as cocaine), 1,2,5-7,12, 15 alongside the recent scientific study and recognition of the extraordinary concentration of deaths in the weeks immediately following release from prison misuse. ${ }^{8,29-34}$

We considered three particular aspects: firstly, the special importance of heroin; secondly, prison as a remarkable concentration of heroin users; and thirdly, the particular concentration in time and context of heroin overdose deaths.

\section{Drug Deaths-the Special Importance of Heroin (and the 0piates)}

Heroin and the opiates contribute disproportionately to drug deaths: even though heroin comprises a minority part of the total use of illicit drugs in the UK, it nevertheless contributes more than $80 \%$ of all drug overdose deaths. ${ }^{26,35}$ Opiates are thus heavily implicated in the attention to deaths as one of the major harms of illicit drug misuse. ${ }^{36-42}$ Many of these opiate overdose deaths also involve simultaneous use of 
other types of drugs (e.g. benzodiazepines and/or alcohol); ${ }^{13,24,36,43-46}$ nevertheless, it is the respiratory depression caused by opiates that is the main mechanism of death. ${ }^{47}$

\section{Concentrations of Heroin Users-Special Concentration in Prisons}

Prisons have an intense, but also complicated, relationship with the heroin-using behavior of so many of their inmates and represent an often overlooked potential intervention opportunity, notwithstanding their primary criminal justice function. ${ }^{48-50}$ While this varies across societies (according to different criminal justice responses to the drug misuse problem), prisons nevertheless comprise an extraordinary concentration of the population at risk of later heroin overdose death. Society achieves, by one means or another, an intense concentration of heroin misusers in its prison population: in the UK, the population lifetime prevalence of less than $1 \%$ amongst the general public is transformed into approximately $40 \%$ in the prison population, both in sentenced populations ${ }^{8,51-55}$ and also in remand populations ${ }^{56-59}$ and has particularly high levels amongst female prisoners. ${ }^{60-62}$ Furthermore, prison can actually be the setting in which heroin use can be initiated. ${ }^{63,64}$ Earlier work by our group identified not only the high pre-incarceration rates of heroin use (at $32 \%$ amongst men) but also the considerable extent to which there was continued use of opiates while still imprisoned ${ }^{50,52}$ —and hence the high probability of re-establishment of heroin use on eventual release.

\section{Concentration of Heroin Overdose Deaths on Release from Prison}

The period following release from prison is a time of extraordinarily high mortality, with heroin overdose deaths concentrated in the first few weeks

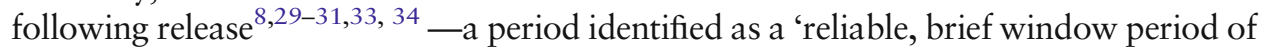
extremely high risk' during which we observe 'carnage among recently released prisoners'. 32 Several studies have now demonstrated this phenomenon in different countries, with risk of overdose death increased more than sevenfold in the first fortnight after release (and remaining significantly elevated in the second fortnight) before gradually subsiding back to heroin users' baseline (but still high) mortality rate. $^{8,29-31,33}$ Of prisoners with a previous history of heroin injecting who are released from prison, an estimated 1 in 200 will die of a heroin overdose within the first 4 weeks of release. Directly comparable figures are hard to find, but this is approximately 10 times the mortality rate of general prisoners being released (who themselves have an increased risk) and approximately 100 times greater than an age-matched general population.

\section{Concentrations of Individuals with Risk Behavior and Concentrations of Deaths in Specific Time: the Special Importance of Prison}

Prisons are thus important for two different reasons. Firstly, society achieves, by one means or another, an extraordinary concentration of heroin users in its prison population. The second particular importance of prison is that the period following release from prison is a time of extraordinarily high mortality, with heroin overdose deaths concentrated in the first few weeks following release.

\section{Considering the Potential for Adverse Reactions}

Concerns about potential unforeseen consequences have been expressed ${ }^{65,66}$ which include the following: that the resuscitation advice may not be heeded or may be applied incorrectly; that there may be possible adverse reactions to naloxone; that 
the resuscitated overdose victim may subsequently drift back into overdose; that blood-borne virus transmission could result from inadvertent needle-stick injury or from re-use. We see these concerns as falling into two major categories that warrant careful attention.

Firstly, the planned intervention may fail to be applied-either not applied at all, or applied insufficiently. There is the possibility that however well-intentioned, the circumstances of the overdose may be such that no one present is appropriately knowledgeable or sufficiently capable to apply the emergency naloxone (and wider resuscitation) intervention. There is also the possibility that there may be technical or operator failure of the naloxone administration. For these reasons, amongst others, we provide the emergency naloxone dose in a pre-filled syringe, in which there is more than adequate dose $(0.8 \mathrm{mg}$ to be given from a pre-filled syringe actually containing $2 \mathrm{mg}$ naloxone), with instructions for it to be given by the much simpler intramuscular route (in contrast to the often intravenous administration by accident and emergency doctors and ambulance paramedics). The pre-filled syringe is given to the N-ALIVE participant in a special general purpose N-ALIVE wallet which not only functions as a regular wallet but also contains essential N-ALIVE information and also the needle and syringe (to be assembled) with the emergency naloxone dose. In the design of the N-ALIVE research study, we have additionally incorporated a post-release telephone interview with a sub-sample (random $50 \%$ of those who consent to telephone follow-up within the first month), and also questionnaire-based re-interview of all N-ALIVE participants who are re-incarcerated to allow us to gather more descriptive information about any overdose events that may have occurred and the emergency use (or loss) of the preprovided naloxone.

A second category of concern is that the very provision of the resuscitation ability may, paradoxically, increase the risk of overdose by virtue of the perception of a 'safety net', provided by the naloxone, which might create a lowered perception of the real risk of fatality and thereby encourage more risky behavior. At least some evidence indicating that this risk may be real can be seen in the response from injecting drug users themselves-amongst whom $6 \%$ indicated, when interviewed about the potential appropriateness of pre-provision of take-home naloxone, that they believed that the provision of such rescue medication may indeed lead them to use more heroin ${ }^{2}$. Prisoners in Barlinnie prison, Glasgow, with whom we discussed this possibility answered frankly that they did not know how they would react.

A third, different type of concern, is that the approach may also be regarded as politically risky, since it could mistakenly be seen to be condoning drug use.

The theoretical possibility of adverse reactions is not sufficient grounds to stand in the way of a randomized effectiveness study if the a priori plausible benefit significantly exceeds the potential harm. The real risk of sudden death needs to be borne in mind while paying proper attention to any evidence that may emerge of adverse effects.

\section{FEASIBILITY OF PROPOSED INTERVENTION}

In the consideration of the N-ALIVE trial, it was necessary for there to be reasonable evidence of the operational feasibility of the proposed intervention-not only that the prescribed emergency supply of opiate anti-dote naloxone would be sufficient to reverse heroin overdose but also that the deliverers of the proposed intervention deliverers (peers and/or family) would not only be likely to be present but also would be willing to be the workforce who deployed the novel intervention. 


\section{The Willingness of Users or Family Members to Intervene}

Those who inject heroin are at high risk of overdose themselves ${ }^{11,43,46,67-69}$ but they are also highly likely to witness the overdose of others-with more than three quarters of injecting heroin users having witnessed an overdose. ${ }^{4,9}, 16,43,67,70-73$ They are thus potentially a vast intervention workforce who are willing to intervene, ${ }^{3,18,69,74}$ but whose actions are currently often inefficient or wrong ${ }^{11,16,73,75,76}$ and who consequently need to be properly trained and provided with the appropriate tools. Furthermore, a quarter of parents at family support groups have already witnessed the overdose of a son or daughter. ${ }^{6}$ In short, a large and well-aware intervention workforce exists.

The extensive willingness of these groups to be actively involved in emergency resuscitation, including the administration of a naloxone injection (if necessary) has now been documented, not only amongst drug users in treatment but also particularly importantly amongst those not currently in treatment ${ }^{2,4,9,18}$ and also amongst carers (e.g. family members). ${ }^{6}$ Indeed, there is clear evidence of active intervention at previous witnessed overdoses, even when the interventions have been the wrong interventions. ${ }^{4,11,16,73,76}$ For example Beswick et al. ${ }^{76}$ found evidence of probably ineffective and potentially harmful interventions, such as additional injection of other drugs (e.g. cocaine), injection of salt, immersion in cold bath, and administering ice. Despite the extent of flawed knowledge, the important conclusion is with regard to the evident strong commitment to intervene amongst fellow injecting drug users and also family members ${ }^{6}$ that can be addressed by training.

To recap, not only has a large potential intervention workforce been identified but also a reported high level of commitment to be taught how to intervene actively. From mostly small-scale pilot naloxone provision schemes, the proportion of distributed naloxone that is used in life-saving interventions is estimated by active clinical teams to be $10 \%{ }^{15,22}$ But, as yet, there have been no definitive randomized trials, with appropriate study design, to establish the extent of benefit (or possible harm) accrued. And it is this scientific evidence vacuum that the N-ALIVE trial addresses.

\section{Naloxone Delivery}

It is likely that the emergency naloxone will need to be administered either by a peer drug user or by a family member, and hence practical decisions were necessary about the most appropriate recommended route of administration, the right dose, and the wider resuscitation management.

Intramuscular injection is an efficient and easily-implemented route of naloxone administration and would appear well-suited to this context. In recent communitybased overdose training for drug users ${ }^{10,14,15,19,21,77}$ and for carers, ${ }^{6}$ the recommended route of administration is intramuscular as this is more suitable for non-specialist administration. Furthermore, even when administered by ambulance personnel, the greater speed with which an intramuscular or subcutaneous injection can be given means that the delay from arrival until recovery of the overdose victim is practically the same as with intravenous administration. ${ }^{78}$

The right dose of naloxone to be provided proved difficult to decide. On the one hand, $0.4 \mathrm{mg}$ is sometimes sufficient, but not always-and the next step would be a repeat dose (i.e. an additional $0.4 \mathrm{mg}$ a few minutes later, thus a total of $0.8 \mathrm{mg}$ ), and, if still no response, a further $0.8 \mathrm{mg}$. Furthermore, in the clinical algorithms used by accident and emergency departments, these dose schedules relate to intravenous administration, and the provision of naloxone by the intramuscular route has a pathway directly to the 0.8-mg initial dose. ${ }^{79}$ Consequently, in the N-ALIVE trial, we provide a pre-prepared 
emergency supply of a single dose of $0.8 \mathrm{mg}$, which is the recommended intramuscular dose in various ambulance and Emergency Medicine protocols in the UK and accords with the clinical algorithm in the Emergency Medical Journal. ${ }^{79}$ Extra doses can then later be given by the emergency medical personnel and/or ambulance staff who subsequently attend the overdose victim.

A possible and simpler means of pre-providing a 0.8 -mg naloxone emergency dose might have been by provision of two 0.4-mg doses (which existed already as commercial formulations), and this solution might be appropriate in community treatment settings, or in a purely observational study. However, in the N-ALIVE trial, this option was not acceptable because of the increased risk of contamination between the experimental and control groups with, for example, 'gift' of one of the naloxone 0.4-mg syringes (from a N-ALIVE participant randomized to the 'naloxone-on-release' intervention) to a fellow release randomized to 'treatment-as-usual'.

Concern has been raised about the relatively short duration of action of naloxone (in the region of an hour or two of main antagonist effect), and the possible drift back into life-threatening overdose (as the naloxone wears off), but this would seem largely ill-founded on the basis of the absence of any deaths recorded over the 5-year study period in San Diego of 998 heroin users who, post-naloxone resuscitation, discharged themselves against medical advice: perhaps some degree of intoxication did occur, but none of these individuals died in the subsequent $12-\mathrm{h}$ period. ${ }^{80,81}$ If it transpired that the relatively shorter duration of action of naloxone (versus heroin, and certainly versus some prescribed pharmaceutical opiates such as methadone) was a valid cause for concern, then either repeat doses may need to be provided (as sometimes provided to recovered overdose victims by ambulance personnel) or alternatively a longer-acting opiate antagonist such as nalmefene could be considered instead. However, given the 40-year history and extensive experience of use of naloxone for reversal of heroin overdose, the N-ALIVE trial is using standard pharmaceutical naloxone.

Naloxone can only save life if it is accessible at the time and place of overdose emergency. Recent surveys of the target population of drug users in treatment suggest that the traditionally provided naloxone is probably only carried 'on the person' by about $20 \%$ of subjects. ${ }^{77}$ We consequently provide an N-ALIVE wallet to each N-ALIVE naloxone participant, in which there is a pouch which contains the naloxone-loaded syringe. For the N-ALIVE trial, information and video demonstration is available on the MRC website at www.ctu.mrc.ac.uk/research_areas/ study_details.aspx?s=80. We also give each N-ALIVE participant a DVD instructional video on essential resuscitation steps and naloxone administration, and the training is also available on YouTube at www.youtube.com/ watch? $\mathrm{v}=\mathrm{Xbnx} 5 \mathrm{Q} 3 \mathrm{vZek}$ in order to explain the N-ALIVE trial itself and also at www.youtube.com/watch? $\mathrm{v}=\mathrm{eMkEndVJi} 7 \mathrm{w} \&$ feature $=$ relmfu to provide instruction on the basics of overdose management and also on potential emergency administration of an injection of naloxone.

\section{DESIGN OF THE N-ALIVE TRIAL}

Many scientific and logistical challenges have been encountered and have needed to be resolved in the design and introduction of the N-ALIVE trial so as to be able to 
quantify the effectiveness (or otherwise) of pre-provision of take-home emergency naloxone and the extent of impact on reducing overdose deaths.

\section{Selection of Randomized Over Observational Trial Design:}

An early crucial decision was whether a randomized trial design (versus observational trial design) was feasible; and, if feasible, whether it was ethical. We consider these two aspects in this section.

The wider provision of pre-placed supply of emergency naloxone has been proposed over more than a decade (first seriously articulated in $1996^{1}$ ) and yet is still the subject of questions about the likelihood of expected genuine benefit, as well as intervention inertia. In such circumstances, and with continued expressions of uncertainty or doubt, the clarity of conclusions from a suitably well-designed and objectively undertaken randomized trial would be a powerful influence on key decision-makers, just as in other contentious domains. ${ }^{28,82}$ However, it is only possible to plan and deliver a randomized trial with participation and consent from the relevant service providers and also crucially from the study population themselves. We have consequently worked closely with the agencies responsible for provision of healthcare in the prison environment (which itself was moved in recent years into the central $\mathrm{NHS}^{83}$ ) to ensure their partnership and support, and we also explored the viability of the proposed trial design with prisoners and ex-prisoners themselves. The high rate of prisoner transfers mitigated against a cluster randomized trial design (including stepped-wedge ${ }^{84,85}$ ) with prison as unit of randomization, and we also wished to incorporate individually informed prisoner consent to randomisation. Consequently, mindful of the continued public and political uncertainty of true effectiveness of naloxone-on-release, and seeking the strongest appropriate trial design for a definitive implementation trial, we proceeded with the opportunity to conduct a full-scale definitive randomized trial.

Ethical issues were also prominent in our consideration, with acute awareness that we might well be providing a life-saving intervention to one group while withholding it from another. In addition to consideration of clinical equipoise, we also needed to consider whether there was policy equipoise. At the time of conducting the study, naloxone is not provided to prisoners on release in England (apart from one prison for women where it had been introduced for a while by an 'early adopter'). Consequently, at all other prisons, there was no withholding from any prisoner on release of any existing treatment or intervention. All prisoners received full offers of participation in education about risk of overdose and, if provided within the particular prison, training in emergency resuscitation. Similarly, no constraints were placed on provision (or nonprovision) of treatments such as methadone or buprenorphine maintenance, or naltrexone-these are all generally approved treatments in the UK, although not always provided as frequently to those with heroin addiction problems within the prison population as would typically be provided in the community context. However, for the trial design, we merely needed to record these other interventions at the time of randomization (by minimization, which takes account of them).

The pre-provision of emergency naloxone remains a contentious proposal in some quarters, and indeed the N-ALIVE trial received challenge on the grounds (mistaken, and misleading) that it involved giving an experimental injectable drug to detoxified prisoners. In fact, the 'experimental' intervention (the pre-provision of a take-home supply of emergency naloxone) involves the provision of a medication developed more than 40 years ago, and being prescribed in an approved form (for intramuscular 
injection) and for the specific purpose for which it is clinically indicated (reversal of opiate overdose): the only novel aspect of the provision was the innovative proposal of pre-placement of the antidote injection with the individual at risk, accompanied by training materials for peers, family and friends via YouTube.

It had originally been planned to conduct the N-ALIVE trial across the UK, but the decision by Fergus Ewing, Scotland's Minister for Community Safety and Legal Affairs, to introduce Scotland-wide provision of naloxone to community and prison release populations at risk of overdose necessitated restriction of the N-ALIVE trial to England (population 52.2 million) since the window of opportunity with policy equipoise had now closed in Scotland (population 5.2 million) (we explore this different development in Scotland in a separate publication-Bird et al., submitted). Additionally, in May 2011, Wales (population 3.0 million) also adopted take-home naloxone as a public health policy.

\section{Selecting the Right Study Sample}

The study sample of current or former heroin injectors on release from a period of imprisonment has been chosen specifically because of their identified excess risk of overdose death. However, even amongst this population, death is a relatively rare event (1 in 200 in first 4 weeks post-release), and hence large numbers of eligible prisonerparticipants need to be randomized to demonstrate as statistically significant a plausible $30 \%$ reduction in overdose deaths. It is scientifically efficient to consider populations in which the event is particularly likely to occur, and at times in their drug-using career when the event may be at increased frequency. Hence, mindful of the marked concentration of overdose deaths in the weeks immediately following release from prison, ${ }^{8,29-31}$ the $\mathrm{N}$ ALIVE trial of take-home emergency naloxone has taken, as its study population, prisoners with a history of heroin injection who are being released from prison.

Using data from the Office of National Statistics (which catalogues every death in the UK), we are then subsequently cross-checking our study sample's identities and release dates against all deaths (in the relevant age-group) in order to identify participants' deaths (and cause of death) in the 12 weeks following release. Comparison of the death rates amongst prisoners randomized to standard health information and after-care versus those randomized to the provision of a take-home emergency supply of naloxone in addition to standard after-care is thus the primary outcome measure of benefit conferred.

\section{The Problem of the Changing Therapeutic Landscape}

There is a shifting baseline in the extent and potential benefits from approved evidence-based treatments, ${ }^{86}$ and also in the recognized importance of healthcare provision in the prison context. ${ }^{87}$ Some of these changes will undoubtedly occur over the course of the N-ALIVE trial, such as improvements in through-care from prison to community (especially continuity of opiate substitution treatment), and the possible introduction of more widespread methadone or buprenorphine maintenance programs as has been commenced in the UK and as has been implemented in other countries. ${ }^{88-91}$ However, the extent of shift of baseline is variable and unpredictable. ${ }^{92}$ Randomization ensures that these changes are affecting equally the two comparison groups in the N-ALIVE trial, and we record changes in these practices while evaluating the extent of benefit from take-home emergency naloxone. Benefit from naloxone may be reduced by more effective continuity of treatment, or the benefit may remain just as pronounced if the overdose deaths are more closely causally related to deliberate post-release excess, rather than just resulting from lack of knowledge about loss of tolerance. Baseline data from 
Scotland (2007-2009) support the latter hypothesis since maintenance treatment in recent years has been readily available and yet $79 / 547(14.4 \%)$ of all Scotland's male opiate-related deaths under the age of 35 still occurred within 4 weeks of prison release, much as in 1996-1999. ${ }^{8}$

\section{The N-ALIVE Trial Starts}

The first subject was recruited into the N-ALIVE randomized trial in May 2012, and trial participants are now being released from prison after having been randomized to either pre-provision of an emergency dose of naloxone (plus information etc.), or alternatively to information but without naloxone supply. This is a significant step forward in the construction of a robust evidence based on how effectively take-home emergency naloxone and training in overdose resuscitation can reduce the extent of overdose deaths in this population.

During this initial pilot phase of the trial, 5,600 prisoners are being randomly assigned to either pre-provision of take-home emergency naloxone, accompanied by explanatory leaflets and instruction for themselves, peers and families, or alternatively to a protocol-driven treatment-as-usual (thus with literature warning of the risk of post-release overdose). Through the full N-ALIVE trial, a total of 56,000 released prisoners will have to be randomized.

This sample size has been calculated on the basis of detecting a $30 \%$ reduction-from the anticipated 140 deaths during the first 4 weeks of release amongst the control group of 28,000 eligible ex-prisoners down to a lower level of just under 100 deaths. N-ALIVE's power calculation is based on various evidence-informed assumptions. If naloxone were universally available, with a competent other always present who had sufficient competence to administer the naloxone, and if properly administered (while also calling ambulance and placing victim in the recovery position, and maintaining assisted breathing etc.), then a survival rate of close to $100 \%$ would presumably be expected for this highly effective opiate anti-dote. However, we are assuming a much more modest penetration of the study population at risk. Since our interest is in real-life application, the preparation for the N-ALIVE trial, and the underlying power calculations, have taken cognizance of the likely real-life application of instructions and hence arrive at a more modest prior estimation of effectiveness. We calculate as follows: we know that as summarized above, approximately $80 \%$ of overdoses occur in the presence of others, and we have conservatively assumed that during the first 4 weeks after release, $75 \%$ of victims will still have their supply of naloxone; we have then assumed that there is perhaps only a 50:50 chance that the other person present will locate the naloxone and have the competence, courage and confidence to administer the intramuscular naloxone. With these assumptions, we thus calculate a $30 \%$ probability of naloxone administration $50 \%$ of $75 \%$ of $80 \%=$ $30 \%$ ), and, when combined with our knowledge of data on mortality rates in the weeks following prison release (summarized above), we identify a necessary sample size of $56,000(28,000$ across each of the two conditions). For fuller details of the power calculation, and also of processes and procedures, the reader is directed to our forthcoming paper on the N-ALIVE trial protocol (in preparation, April 2013).

\section{CONCLUSION: DRUG-RELATED DEATHS, MISSED OPPORTUNITIES AND THE APPLIANCE OF SCIENCE}

The start of the N-ALIVE trial is a highly significant scientific step in the development of an evidence-based harm reduction initiative to stem the hemorrhage of heroin overdose 
deaths that has been identified as occurring in the period immediately post-prison release. The results from the N-ALIVE trial will also have profound importance for the planning and provision of more effective responses sight across the addiction treatment field, and particularly with regard to the community prevention of the marked excess mortality of heroin users after other high-risk discharges, such as from hospital. ${ }^{93}$ The N-ALIVE trial stands as an examplar research study selected and designed to give a definitive answer to society at large, politicians and policymakers, as well as to clinicians and researchers, with results likely to have wide generalizable significance.

Advocates call for action, while naysayers raise concerns. N-ALIVE will test this specific proposed method of reducing the overdose death carnage post-prison release; it will give us a robust evidence base on which to make important decisions; and it will increase our understanding of the interacting relationships between drugtaking behavior and associated health hazards, the application of criminal justice sanctions, and the provision of health interventions. Wider provision of naloxone has now been proposed (and opposed) for more than a decade. The issue does not need more public debate; it now needs good science.

\section{ACKNOWLEDGMENTS}

Our particular thanks are due to Julie Bakobaki, Susie Hennings, Angela Meade and Nicola Muirhead for assistance with development work, to Dave Marteau and Nino Maddalena (English Prison Health Care service) and Karen Norrie, Ruth Parker and Stephen Heller-Murphy (Scottish Prison Health Care service) and Dr. Andrew Fraser (formerly head of healthcare at SPS), to the prisoners who gave generously of the opinions and advice, and to the MRC (Medical Research Council) who are supporting the trial.

Open Access This article is distributed under the terms of the Creative Commons Attribution License which permits any use, distribution, and reproduction in any medium, provided the original author(s) and the source are credited.

\section{REFERENCES}

1. Strang J, Darke S, Hall W, Farrell M, Ali R. Heroin overdose: the case for take-home naloxone. BMJ. 1996; 312: 1435-1436.

2. Strang J, Powis B, Best D, et al. Preventing opiate overdose fatalities with take-home naloxone: pre-launch study of possible impact and acceptability. Addiction. 1999; 94: 199-204.

3. Strang J. Take-home naloxone: the next steps. Comments on Strang et al'.s 'Preventing opiate overdose fatalities with take-home naloxone: pre-launch study of possible impact and acceptability'. Addiction. 1999; 94: 206-207.

4. Best D, Gossop M, Man LH, Stillwell G, Coomber R, Strang J. Peer overdose resuscitation: multiple intervention strategies and time to response by drug users who witness overdose. Drug Alcohol Rev. 2002; 21: 269-274.

5. Strang J, Kelleher MJ, Best D, Mayet S, Manning V. Preventing heroin overdose deaths with emergency naloxone. Br Med J. 2006; 333: 614-615.

6. Strang J, Manning V, Mayet S, et al. Family carers and the prevention of heroin overdose deaths: unmet training need and overlooked intervention opportunity. Drugs Educ Prev Policy. 2008; 15: 211-218.

7. Darke S, Hall W. The distribution of naloxone to heroin users. Addiction. 1997; 92: 1195-1199.

8. Bird SM, Hutchinson SJ. Male drugs-related deaths in the fortnight after release from prison: Scotland, 1996-99. Addiction. 2003; 98: 185-190. 
9. Seal KH, Downing M, Kral AH, et al. Attitudes about prescribing take-home naloxone to injection drug users for the management of heroin overdose: a survey of street-recruited injectors in the San Francisco Bay Area. J Urban Health. 2003; 80: 291-301.

10. Seal KH, Thawley R, Gee L, et al. Naloxone distribution and cardiopulmonary resuscitation training for injection drug users to prevent heroin overdose death. J Urban Health. 2005; 82: 303-311.

11. Tracy M, Piper TM, Ompad D, et al. Circumstances of witnessed drug overdose in New York City: implications for intervention. Drug Alcohol Depend. 2005; 79: 181-190.

12. Baca CT, Grant KJ. Take-home naloxone to reduce heroin death. Addiction. 2005; 100: 1823-1831.

13. Coffin PO, Galea S, Ahern J, Leon AC, Vlahov D, Tardiff K. Opiates, cocaine and alcohol combinations in accidental drug overdose deaths in New York City, 1990-98. Addiction. 2003; 98: 739-747.

14. Galea S, Worthington N, Piper TM, Nandi VV, Curtis M, Rosenthal DM. Provision of naloxone to injection drug users as an overdose prevention strategy: early evidence from a pilot study in New York City. Addict Behav. 2006; 31: 907-912.

15. Maxwell S, Bigg D, Stanczykiewicz K, Carlberg-Racich S. Prescribing naloxone to actively injecting heroin users: a program to reduce heroin overdose deaths. J Addict Dis. 2006; 25: 89-96.

16. Pollini RA, McCall L, Mehta SH, Celentano DD, Vlahov D, Strathdee SA. Response to overdose among injection drug users. Am J Prev Med. 2006; 31: 261-264.

17. Wright N, Oldham N, Francis K, Jones L. Homeless drug users' awareness and risk perception of peer "Take Home Naloxone" use-a qualitative study. Subst Abuse Treat Prev Pol. 2006; 1: 28.

18. Lagu T, Anderson BJ, Stein M. Overdoses among friends: drug users are willing to administer naloxone to others. J Subst Abuse Treat. 2006; 30: 129-133.

19. Worthington N, Markham PT, Galea S, Rosenthal D. Opiate users' knowledge about overdose prevention and naloxone in New York City: a focus group study. Harm Reduct J. 2006; 3: 19.

20. Sporer KA, Kral AH. Prescription naloxone: a novel approach to heroin overdose prevention. Ann Emerg Med. 2007; 49: 172-177.

21. Sherman S, Gann D, Scott G, Carlberg S, Bigg D, Heimer R. A qualitative study of overdose responses among Chicago IDUs. Harm Reduction J. 2008; 5: 2.

22. Dettmer K, Saunders B, Strang J. Take home naloxone and the prevention of deaths from opiate overdose: two pilot schemes. BMJ. 2001; 322: 895-896.

23. Bigg D. Data on take-home naloxone are unclear but not condemnatory. BMJ. 2002; 324: 678 .

24. Galea S, Nandi A, Coffin PO, et al. Heroin and cocaine dependence and the risk of accidental non-fatal drug overdose. J Addict Dis. 2006; 25: 79-87.

25. Markham PT, Rudenstine S, Stancliff S, et al. Overdose prevention for injection drug users: lessons learned from naloxone training and distribution programs in New York City. Harm Reduct J. 2007; 4: 3.

26. Advisory Council on the Misuse of Drugs (ACMD). Drug-related deaths. London: Home Office. London, England: The Stationery Office; 2000.

27. Advisory Council on the Misuse of Drugs (ACMD). Consideration of naloxone. London: Home Office. London, England: The Stationery Office; 2012.

28. Edwards G, Strang J, Jaffe JH. Drugs alcohol tobacco: making the science and policy connections. Oxford, England: Oxford University Press; 1993.

29. Seaman SR, Brettle RP, Gore SM. Mortality from overdose among injecting drug users recently released from prison: database linkage study. BMJ. 1998; 316: 426428.

30. Binswanger IA, Stern MF, Deyo RA, et al. Release from prison-a high risk of death for former inmates. N Engl J Med. 2007; 356: 157-165.

31. Farrell M, Marsden J. Acute risk of drug-related death among newly released prisoners in England and Wales. Addiction. 2008; 103: 251-255. 
32. Darke S. [Commentary] From the can to the coffin: deaths among recently released prisoners. Addiction. 2008; 103: 256-257.

33. Merrall E, Kariminia A, Binswanger IA, Hobbs M, Farrell M, Marsden J, Hutchinson SJ, Bird SM. Meta-analysis of drug-related deaths soon after release from prison. Addiction. 2010; 105: 1545-1554.

34. Kariminia A, Butler T, Corben S, Levy M, Grant L, Kaldor J, et al. Extreme cause-specific mortality in a cohort of adult prisoners-1988 to 2002: a data-linkage study. Int J Epidemiology. 2007; 36: 310-316.

35. HM Government. Drugs: protecting families and communities. The 2008 Drug Strategy. Central Office of Information on behalf of HM Government, 2008. Report No.: CDSD9.

36. Darke S, Zador D. Fatal heroin 'overdose': a review. Addiction. 1996; 91: 1765-1772.

37. Hickman M, Madden P, Henry J, et al. Trends in drug overdose deaths in England and Wales 1993-98: methadone does not kill more people than heroin. (Erratum appears in Addiction 98: 1325-6). Addiction. 2003; 98: 419-425.

38. Davidson P, McLean R, Kral A, Gleghorn A, Edlin B, Moss A. Fatal heroin-related overdose in San Francisco, 1997-2000: a case for targeted intervention. J Urban Health. 2003; 80: 261-273.

39. Bryant WK, Galea S, Tracy M, Markham Piper T, Tardiff KJ, Vlahov D. Overdose deaths attributed to methadone and heroin in New York City, 1990-1998. Addiction. 2004; 99: 846-854.

40. Morgan OW, Johnson H, Rooney C, Seagroatt V, Griffiths C. Changes to the daily pattern of methadone-related deaths in England and Wales, 1993-2003. J Public Health. 2006; 28: 318-323.

41. Davoli M, Bargagli AM, Perucci CA, et al. Risk of fatal overdose during and after specialist drug treatment: the VEdeTTE study, a national multi-site prospective cohort study. Addiction. 2007; 102: 1954-1959.

42. Bird S. Shellacking over drug harms (response to Nutt, et al. Drug Harm in the UK). Lancet. 2010; 377: 552-553.

43. Powis B, Strang J, Griffiths P, et al. Self-reported overdose among injecting drug users in London: extent and nature of the problem. Addiction. 1999; 94: 471-478.

44. Strang J, Best D, Man LH, Noble A, Gossop M. Peer-initiated overdose resuscitation: fellow drug users could be mobilised to implement resuscitation. Int J Drug Policy. 2000; 11: 437-445.

45. Hickman M, Carrivick S, Paterson S, et al. London audit of drug-related overdose deaths: characteristics and typology, and implications for prevention and monitoring. Addiction. 2007; 102: 317-323.

46. Coffin PO, Tracy M, Bucciarelli A, Ompad D, Vlahov D, Galea S. Identifying injection drug users at risk of nonfatal overdose. Acad Emerg Med. 2007; 14: 616-623.

47. White JM, Irvine RJ. Mechanisms of fatal opioid overdose. Addiction. 1999; 94: 961-972.

48. Strang J. Sexual and injecting behaviors of prisoners: from disciplinary problem to public health conundrum. Crim Behav Ment Health. 1993; 3: 393-402.

49. Bird AG, Gore SM, Burns SM, Duggie JG. Study of infection with HIV and related risk factors in young offenders' institution. BMJ. 1993; 307: 228-231.

50. Bird AG, Gore SM, Hutchinson SJ, Lewis SC, Cameron S, Burns S. Harm reduction measures and injecting inside prison versus mandatory drugs testing: results of a cross sectional anonymous questionnaire survey. The European Commission Network on HIV infection and hepatitis in prison. BMJ. 1997; 315: 21-24.

51. Maden A, Swinton M, Gunn J. A survey of pre-arrest drug use in sentenced prisoners. Addiction. 1992; 87: 27-33.

52. Strang J, Gossop M, Heuston J, Green J, Whiteley C, Maden A. Persistence of drug use during imprisonment: relationship of drug type, recency of use and severity of dependence to use of heroin, cocaine and amphetamine in prison. Addiction. 2006; 101: 11251132. 
53. Fazel S, Bains P, Doll H. Substance abuse and dependence in prisoners: a systematic review. Addiction. 2006; 101: 181-191.

54. Rounds-Bryant JL, Baker L. Substance dependence and level of treatment need among recently-incarcerated prisoners. Am J Drug Alcohol Abuse. 2007; 33: 557-561.

55. Gore SM, Bird AG. Study size and documentation to detect injection-related hepatitis C in prison. QJM. 1998; 91(5): 353-357.

56. Mason D, Birmingham L, Grubin D. Substance use in remand prisoners: a consecutive case study. BMJ. 1997; 315: 18-21.

57. Brooke D, Taylor C, Gunn J, Maden A. Substance misusers remanded to prison-a treatment opportunity? Addiction. 1998; 93: 1851-1856.

58. Brooke D, Taylor C, Gunn J, Maden A. Substance misuse as a marker of vulnerability among male prisoners on remand. Br J Psychiatry. 2000; 177: 248-251.

59. Farrell M, Boys A, Singleton N, et al. Predictors of mental health service utilization in the 12 months before imprisonment: analysis of results from a national prisons survey. Aust N Z J Psychiatry. 2006; 40: 548-553.

60. Maden A, Swinton M, Gunn J. Women in prison and use of illicit drugs before arrest. BMJ. 1990; 301: 1133.

61. Gore SM, Bird GA, Burns S, Ross AJ, Goldberg D. Anonymous HIV surveillance with risk-factor elicitation: at Perth (for men) and Cornton Vale (for women) Prisons in Scotland. Int J STD AIDS. 1997; 8: 166-175.

62. Maden T, Swinton M, Gunn J. Psychiatric disorder in women serving a prison sentence. Br J Psychiatry. 1994; 164: 44-54.

63. Boys A, Farrell M, Bebbington P, et al. Drug use and initiation in prison: results from a national prison survey in England and Wales. Addiction. 2002; 97: 1551-1560.

64. Gore SM, Bird AG, Ross AJ. Prison rites: starting to inject inside. BMJ. 1995; 311: 1135-1136.

65. Lenton SR, Hargreaves KM. Should we conduct a trial of distributing naloxone to heroin users for peer administration to prevent fatal overdose? Med J Aust. 2000; 173: 260-263.

66. Mountain D. Big conclusions are drawn from little evidence. BMJ. 2001; 323: 934.

67. Darke S, Ross J, Hall W. Overdose among heroin users in Sydney, Australia: II. Responses to overdose. Addiction. 1996; 91: 413-417.

68. Darke S, Williamson A, Ross J, Teesson M. Non-fatal heroin overdose, treatment exposure and client characteristics: findings from the Australian Treatment Outcome Study (ATOS). Drug Alcohol Rev. 2005; 24: 425-432.

69. Coffin PO, Fuller C, Vadnai L, Blaney S, Galea S, Vlahov D. Preliminary evidence of health care provider support for naloxone prescription as overdose fatality prevention strategy in New York City. J Urban Health. 2003; 80: 288-290.

70. Gossop M, Griffiths P, Powis B, Williamson S, Strang J. Frequency of non-fatal heroin overdose: survey of heroin users recruited in non-clinical settings. BMJ. 1996; 313: 402.

71. McGregor C, Darke S, Ali R, Christie P. Experience of non-fatal overdose among heroin users in Adelaide, Australia: circumstances and risk perceptions. Addiction. 1998; 93: 701-711.

72. Bennett GA, Higgins DS. Accidental overdose among injecting drug users in Dorset, UK. Addiction. 1999; 94: 1179-1189.

73. Davidson PJ, Ochoa KC, Hahn JA, Evans JL, Moss AR. Witnessing heroin-related overdoses: the experiences of young injectors in San Francisco. Addiction. 2002; 97: 1511-1516.

74. Baca CT, Grant KJ. What heroin users tell us about overdose. J Addict Dis. 2007; 26: 63-68.

75. Best D, Gossop M, Man L-H, Finch E, Greenwood J, Strang J. Accidental and deliberate overdose among opiate addicts in methadone maintenance treatment: are deliberate overdoses systematically different? Drug Alcohol Rev. 2000; 19: 213-216.

76. Beswick T, Best D, Bearn J, et al. From salt injection to naloxone: accuracy and myths in peer resuscitation methods for opiate overdose. J Drug Issues. 2002; 32: 1103-1114. 
77. Strang J, Manning V, Mayet S, Best D, Titherington E, Santana L. Preventing overdose death through overdose management training and provision of take-home naloxone to opiate users in treatment: prospective cohort study of impact on knowledge, attitudes and behavior. Addiction. 2008; 103: 1648-1657.

78. Wanger K, Brough L, Macmillan I, Goulding J, MacPhail I, Christenson J. Intravenous vs subcutaneous naloxone for out-of-hospital management of presumed opioid overdose. Acad Emerg Med. 1998; 5: 293-299.

79. Clarke SF, Dargan PI, Jones AL. Naloxone in opioid poisoning: walking the tightrope. Emerg Med J. 2005; 22: 612-616.

80. Vilke GM, Buchanan J, Dunford JV, Chan TC. Are heroin overdose deaths related to patient release after pre-hospital treatment with naloxone? Prehosp Emerg Care. 1999; 3: 183-186.

81. Vilke GM, Sloane C, Smith AM, Chan TC. Assessment for deaths in out-of-hospital heroin overdose patients treated with naloxone who refuse transport. Acad Emerg Med. 2003; 10: 893-896.

82. Strang J, Metrebian N, Lintzeris N, Potts L, Carnwarth T, Mayet S, et al. Heroin on trial: the RIOTT randomized trial of supervised injectable heroin and injectable methadone as treatment for chronic heroin addicts persistently failing in orthodox treatment. Lancet. 2010; 375: 1885-1895.

83. Mackie P, Morling J. Commissioning prison health: opportunities and challenges for creating a new prison public health system in Scotland. Public Health. 2009; 123: 434-437.

84. Brown CA, Lilford RJ. The stepped wedge trial design: a systematic review. BMC Med Res Methodol. 2006; 6: 54.

85. Hussey MA, Hughes JP. Design and analysis of stepped wedge cluster randomized trials. Contemp Clin Trials. 2007; 28: 182-191.

86. Strang J, Babor T, Caulkins J, Fischer B, Foxcroft D, Humphreys K. Drug policy and the public good: evidence for effective interventions. Lancet. 2012; 379: 71-83.

87. Reed J, Lyne M. The quality of health care in prison: results of a year's programme of semi-structured inspections. BMJ. 1997; 315: 1420-1424.

88. Dolan K, Wodak AD, Hall WD. Methadone maintenance treatment reduces heroin injection in New South Wales prisons. Drug Alcohol Rev. 1998; 17: 153-158.

89. Dolan KA, Shearer J, MacDonald M, Mattick RP, Hall W, Wodak AD. A randomized controlled trial of methadone maintenance treatment versus wait list control in an Australian prison system. Drug Alcohol Depend. 2003; 72: 59-65.

90. Whitling NJ. New policy on methadone maintenance treatment in prisons established in Alberta. Can HIV AIDS Policy Law Rev. 2003; 8: 45-47.

91. Kinlock TW, Gordon MS, Schwartz RP, O’Grady K, Fitzgerald TT, Wilson M. A randomized clinical trial of methadone maintenance for prisoners: results at 1-month post-release. Drug Alcohol Depend. 2007; 91: 220-227.

92. Rich J, Boutwell A, Shield D, et al. Attitudes and practices regarding the use of methadone in US State and federal prisons. J Urban Health. 2005; 82: 411-419.

93. Merrall EL, Bird SM, Hutchinson SJ. A record-linkage study of drug-related death and suicide after hospital discharge among drug-treatment clients in Scotland, 1996-2006. Addiction. 2012. doi:10.1111/j.1360-0443.2012.04066 